Dermatologische Zeitschrift. 1902;9:601-602

\title{
Eine neue Japanische Zeitschrift
}

Mit besonderer Grenugthuu $\pi \mathrm{g}$ begriissen wir die Japanisehe Zeitschrift für Dermatologie und Urologie, welche von Prof. Dr. K. Dolii-Tokio in G- em ein sch aft mit Dr. T. Okamura 602 Tagesnachrichten und Notizcn. - Personalien.

neuei'diogs herausgegeben wird. Jährlich erscheinen fünf bis sechs Hefte, von denen die ersten fünf mit stattlichen Abbildungen versehen vor uns liegen. Dieselben enthalten Beiträge:

Zur Kenntniss der systematisierten Naevi. Yon Dr. Okamura.

Ueber die Lepra in Japan. Yon Prof. K. Do hi.

3. Ueber die Antipyrinexantheme. Yon Stabsarzt Dr. Yamada.

4. Ueber die Verwendung von Röntgenstrahlen in der Dermato-

tlierapie. Yon Dr. Okamura.

Ueber Nierentuberkulose. Von S. Koniaya.

Ueber den Blutbefu $\pi$ d bei Leprakranken. Von Dr. Takasu.

Ueber Venerie bei Civil- und Militärpersonen. Von Stabsarzt

Dr. Ashiliara.

Aus alledem geht hervor, dass unsere japanischen Special-kollegen sicli in ungemein reger

Weise an der Ausarbeitung des dermatologischen Forschungsgebietes betheiligen und unter der Leitung ihres gelehrten Führers Dohi einen selbstänciigen Platz in der Originallitteratur einzunehmen beginnen. Yvir beglück-wünschen unsere ostasiatischen Freunde zu ihrem Vorgehen und werden beständig ihren Veröffentlichungen mit grosser Auf-merksamkeit folgen. Auf den Inbalt der erwähnten Arbeiten kommen wir im Abschnitt ,periodische Litteratur” des nächsten Heftes zurück.

Personalien.

Ais Nachfulger Moriz Kaposi's ist Hen- Professor Dr. Riehl in Leipzig zum ordentliclien Professor der Dermatologie an der Wiener Uni-versität ernannt worden.

In Breslau hat sicli Dr. V. Klingmüller, Oberarzt der Dermatologischen Universitätsklinik, als Privatdozent für Dermatologie habilitirt.

TJnser lieber und geschätzter Mitarbeiter, ein getreuer Schüler des Herausgebers dieser Zeitschrift, der vortrefflíehe Bearbeiter der holländisch-indischen Lepra, Dr. Broes van Doort in Rotterdam, ist seínen zahlreiehen Freanden und Patient $\beta$ n naeh kurzem Leiden unerwartet durch den Tod eutrissen worden. Seinern Andenken sei Ehre! 